\title{
CCD astrolabe solar diameter measurements in 1999-2000
}

\author{
J. L. Penna ${ }^{1}$, E. G. Jilinski ${ }^{1,2,3}$, A. H. Andrei ${ }^{1}$, S. P. Puliaev ${ }^{3}$, and E. Reis Neto ${ }^{1}$ \\ 1 Observatório Nacional, MCT, R. Gal. José Cristino 77, Rio de Janeiro, Brasil \\ 2 Laboratório Nacional de Computação Científica, MCT, Brasil \\ 3 Pulkovo Observatory, St. Peterburg, Russia \\ Received 15 October 2001 / Accepted 4 December 2001
}

\begin{abstract}
Solar diameter observations are presented covering a period close to the maximum of the solar activity cycle 23, from July 1999 to December 2000, at the Observatório Nacional, Rio de Janeiro. The observations were made at $\lambda=5635 \AA$, with a CCD Solar Astrolabe, thus timing the limb crossing through a fixed zenith distance. The formal precision of a single measurement is typically 0 ". 18 . The results represent daily means from 4625 independent measurements. The series obtained has 225 homogeneously distributed points with a mean precision of 0. ' 12 . The average value obtained for the solar semi-diameter within the analyzed period is $9599^{\prime \prime} 01 \pm 0$.' 02 . The average of the observations, made under a wide range of different geometric, instrumental and environmental conditions, aims to provide results representing global variations of the solar radius. From this data, variations with amplitude equal to or larger than 0 '. 02 can be investigated. No statistically significant linear trend is obtained for the examined series. There is, however, some indication of an increasing trend for 1999 followed by a stabilization during 2000. Periodic variations are obtained with amplitudes between 0. . 02 and 0. . 07 . Periods related to the solar rotation and the sunspot characteristic lifetime can be recognized.
\end{abstract}

Key words. Sun: activity - Sun: fundamental parameters

\section{Introduction}

Time variations in the solar radius have been discussed by several authors (Laclare et al. 1999). The causes, timing and amplitude, however, are still subjects of investigation. Astrometric solar observations can be affected by atmospheric conditions (and their variation), causing errors on the measured semi-diameter, varying from 0.11 to $1^{\prime \prime}$ for a single measurement (Irbah et al. 1994). On a smaller scale, variations of the solar figure, including the solar oblateness, can introduce discrepancies of the order of $0 . \prime 01$ in the radius measured along different heliolatitudes (Khun et al. 2000). Both difficulties can be overcome by taking a large number of observations, covering different atmospheric conditions and pointing over a substantial range of local directions, as well as directions around the solar figure.

At the Observatório Nacional station (ON, $\phi=$ $-22^{\circ} 53^{\prime} 42^{\prime \prime}, \Lambda=+2^{\mathrm{h}} 52^{\mathrm{m}} 53.5, h=33 \mathrm{~m}$ ), the observations are made using a modified solar astrolabe, equipped with a variable angle frontal reflecting prism. The observations started in 1997, and the full account of the observational settings and the analysis of the first results have been presented elsewhere (Jilinski et al. 1998, 1999). The zenith distance observational range goes from $25^{\circ}$ to $55^{\circ}$. Up to 50 independent measurements can be taken daily,

Send offprint requests to: J. L. Penna,

e-mail: jucira@on.br covering a wide interval of heliolatitudes. Also, the observations span the whole apparent solar orbit, avoiding systematic gaps in the series. Further, since East and West sessions are held, symmetric azimuths are sighted from the meridian and a variety of atmospheric conditions are encountered, this helps to prevent systematic effects on the daily average measured solar diameter.

Here we report the results obtained during the period from 1999.5 to 2001.0. This period covers the maximum of the solar activity for cycle 23 . The investigation aims to assess variations on large scales across the observed solar limb. In the next section, the observed series is presented. The statistical analysis focuses on the errors of the daily average semi-diameter, in order to qualify the results that can be obtained from it. Section 3 discusses whether the observational conditions introduce biases on the results, and whether systematic variations of the observed solar diameter can be recognized in the series. The main aspects and conclusions of this work are summarized in the last section.

Since faulty measurements were searched for and subsequently corrected or removed, these results supersede all others based on preliminary data from this program.

\section{The observed series}

The series here presented starts in July/1999 until the end of December/2000. Previous results have already been 
presented (Jilinski et al. 1999; Puliaev et al. 2000). The observations are centered at $\lambda 5635 \AA$, with an effective bandpass of $\lambda=1680 \AA$. During this period, 5031 individual measurements were taken. Prior to the analysis, 119 measurements were discarded because they lay beyond a threshold of three times the standard deviation for the whole series, 272 measurements were removed because of faulty orientation of the CCD camera. In addition, 15 measurements were abandoned, having been made in sessions (either East or West) in which less than three independent measurements were taken. Due to this criterion, 3 observing days were finaly abandoned. The remaining 4625 individual measurements have average formal precision of 0 ". 18 and average accuracy of 0.51 . No corrections were applied to the measurements other than those from the software treatment (Sinceac 1999). They were combined to produce the series of 225 mean daily semi-diameters represented in Fig. 1. The individual values displayed are given in a table ${ }^{1}$ available only in electronic form. It contains:

- year, month and day of the observation;

- the modified julian date;

- the number of independent measurements;

- the average semi-diameter;

- the standard deviation for the daily measurements;

- the range of observed zenith distances.

Table 1 shows the monthly averages of the measured semi-diameter.

The average number of observations is 20.6 per day $(\sigma=9.0 \mathrm{obs} /$ day). These observations are taken under a variety of atmospheric conditions ( $\Delta T$ average range is $\left.6.7^{\circ} \mathrm{C}, \sigma=1.9^{\circ} \mathrm{C}\right)$, zenith distances $(\Delta z$ average range is $10.9^{\circ}, \sigma=4.1^{\circ}$ ), heliolatitudes (heliolatitude average range of $31.0^{\circ}, \sigma=23.8^{\circ}$ ), and azimuths ( $\Delta A$ average range of $13.8^{\circ}, \sigma=8.4^{\circ}$ ), in the latter case grouping east and west observations along the same direction.

The correlation between the semi diameter scattering distribution and a normal distribution is larger than $95 \%$. This indicates that the large number of observations and the variety of observational conditions were capable of producing a series without systematic effects. In particular the observations along many directions of heliolatitude allow us to probe overall variations of the solar semi-diameter, as the discrepancies due to the solar figure are at the $0^{\prime \prime} 01$ level. The precision of a single daily average measured semi-diameter is $0^{\prime \prime} 12$. Since the series has 225 points, systematic variations larger than 0 .'008 can, in principle, be searched for.

However, although dense, the data are unequally spaced. To investigate the consequence of such inequality, white noise fake data, with the standard deviation taken from the series, was analyzed from 100 Monte Carlo simulations. The analyses show 0 ".02 as the maximum amplitude of periodic terms that could be introduced. This

1 Available only in electronic form at the CDS via anonymous ftp to cdsarc.u-strasbg.fr $(130.79 .128 .5)$ or via http://cdsweb.u-strasbg.fr/cgi-bin/qcat?J/A+A/384/650
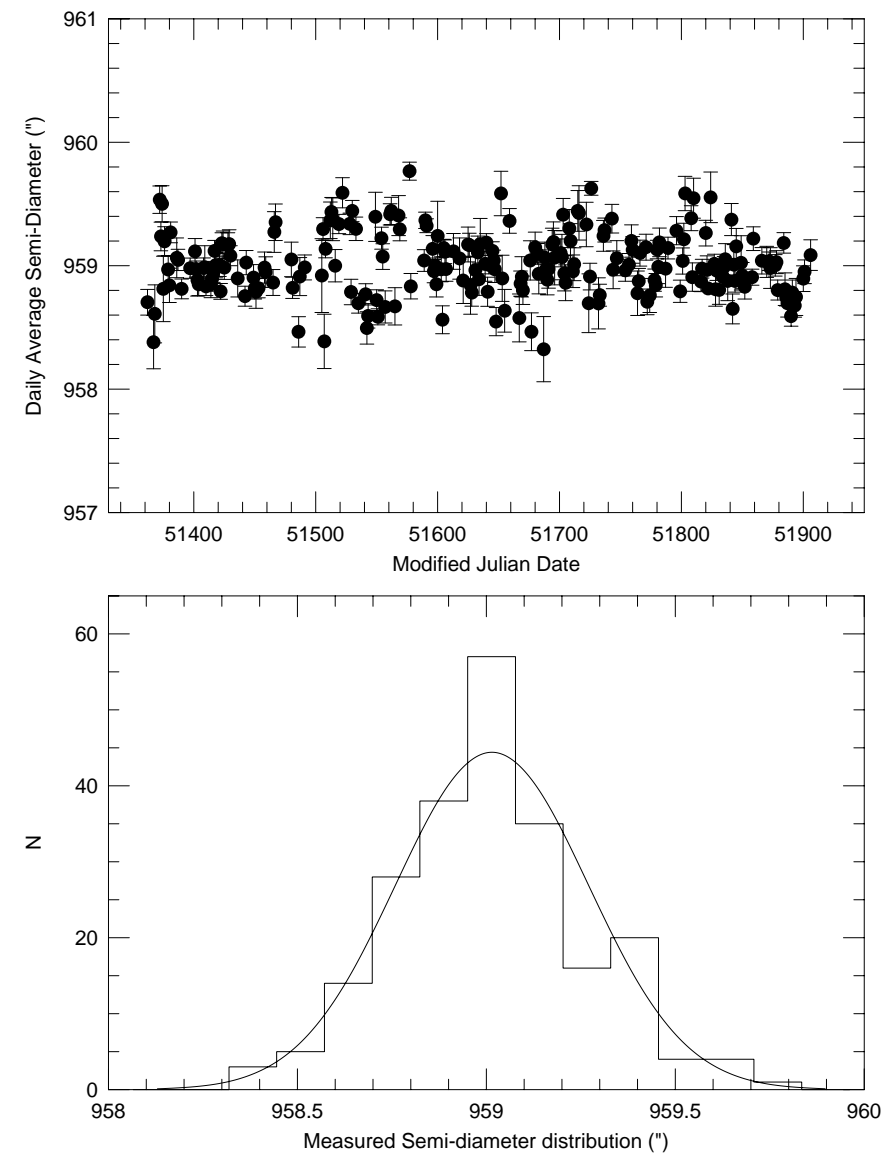

Fig. 1. On the top plot the series of daily average solar semidiameter. On the botton the histogram of the daily values scatter around the mean value (superimposed with a Gaussian curve of same variance).

value is then assumed as the minimum level of significance for the results.

\section{Results}

In Figs. 2-5, the series data and associated errors are displayed as a function of the number of observations, azimuth, zenith distance and temperature. The inspection of the plots shows no significant systematic effects on the observed semi-diameter. Concerning the error on the average daily semi-diameter, only dependencies with the number of observations and with the zenith distance are seen. Least squares polynomial fits, from first to third degrees, of the semi-diameter against the number of observations, or azimuth, or zenith distance or temperature show no statistically significant dependence, nor do the adjustements diminish the standard deviation of the series. As for the error on the average daily semi-diameter, a linear dependence with increasing zenith distance ( 0.3 mas/degree) is found; likewise an inverse square root dependence with the number of observations is seen. In Fig. 5, there is a suggestion of large semi-diameter standard errors for the cooler temperatures; the least-squares adjustments, however, do not show any significant dependence. 
Table 1. Monthly average observed solar semi-diameter (SD). In the table, " $N$ " is the number of observed days in the month; "Err" is the semi-diameter error and " $\sigma$ " is the standard desviation for the monthly distribution.

\begin{tabular}{|c|c|c|c|c|c|}
\hline$N$ & SD $\left(^{\prime \prime}\right)$ & Err $\left(^{\prime \prime}\right)$ & $\sigma\left(^{\prime \prime}\right)$ & Month & Year \\
\hline 14 & 959.00 & 0.09 & 0.32 & Jul. & 1999 \\
15 & 958.95 & 0.02 & 0.09 & Aug. & 1999 \\
12 & 959.00 & 0.05 & 0.16 & Sep. & 1999 \\
8 & 959.01 & 0.07 & 0.19 & Oct. & 1999 \\
9 & 958.99 & 0.12 & 0.35 & Nov. & 1999 \\
12 & 959.06 & 0.10 & 0.36 & Dec. & 1999 \\
11 & 959.08 & 0.10 & 0.33 & Jan. & 2000 \\
12 & 959.09 & 0.09 & 0.30 & Feb. & 2000 \\
14 & 959.03 & 0.04 & 0.13 & Mar. & 2000 \\
12 & 959.01 & 0.08 & 0.28 & Apr. & 2000 \\
17 & 958.91 & 0.06 & 0.24 & May & 2000 \\
15 & 959.15 & 0.07 & 0.26 & Jun. & 2000 \\
9 & 959.04 & 0.07 & 0.22 & Jul. & 2000 \\
14 & 958.98 & 0.04 & 0.17 & Aug. & 2000 \\
11 & 959.16 & 0.08 & 0.26 & Sep. & 2000 \\
18 & 959.01 & 0.05 & 0.21 & Oct. & 2000 \\
11 & 958.98 & 0.03 & 0.11 & Nov. & 2000 \\
11 & 958.83 & 0.05 & 0.17 & Dec. & 2000 \\
\hline
\end{tabular}

The average value for the solar semi-diameter obtained for this series is $959^{\prime \prime} 01 \pm 00^{\prime \prime} 02(\sigma=0.25)$. The average standard error for the daily value is $00^{\prime \prime} 12$. Since no systematic observational error was found, a search was made for time variations of the solar radius. The value for the average semi-diameter agrees well with that obtained by the solar astrolabe at Tubitak (Golbasi et al. 2001), namely $959^{\prime \prime} 03$. It is however smaller than that from the Calern observations (Pap et al. 2001) and from Santiago (Noel 2001), respectively 959'.42 and 960".37. Differences between the threshold of detection of CCD cameras and the human eye, as well as between the observer's judgment of the solar limb boundary as compared to that from the CCD image treatment could qualitatively explain the discrepancies. Still, the Calern experience of side by side work of visual and CCD solar astrolabes has shown that the difference in averages does not mean different observed variations of the semi-diameter (Chollet \& Sinceac 1999).

A least squares linear adjustment of the observed semidiameter with time shows no trend above $0^{\prime \prime} 008 /$ yr for the entire period studied. However, a second degree polynomial least squares fitting suggests a tendency towards a downward concavity. This result is supported when separate linear adjustments are made for the years 1999 and 2000. For 1999 , the linear trend is $+0^{\prime \prime} .1 / \mathrm{yr} \pm 0$. . $05 / \mathrm{yr}$, while for 2000 it is $-0{ }^{\prime \prime} 1 / \mathrm{yr} \pm 00^{\prime \prime} 07 / \mathrm{yr}$. No time dependence is found for the standard error.

A CLEAN algorithm (Roberts et al. 1987) was used to detect periodicities in the data. No periodic variations are found either in the accuracy or in the precision. Table 2 shows the outcome of the CLEAN analysis regarding the semi-diameter. Periods tied to the length of the series and those tied to the Nyquist frequency (10 days), although detected, are discarded. Within the periods kept, those of
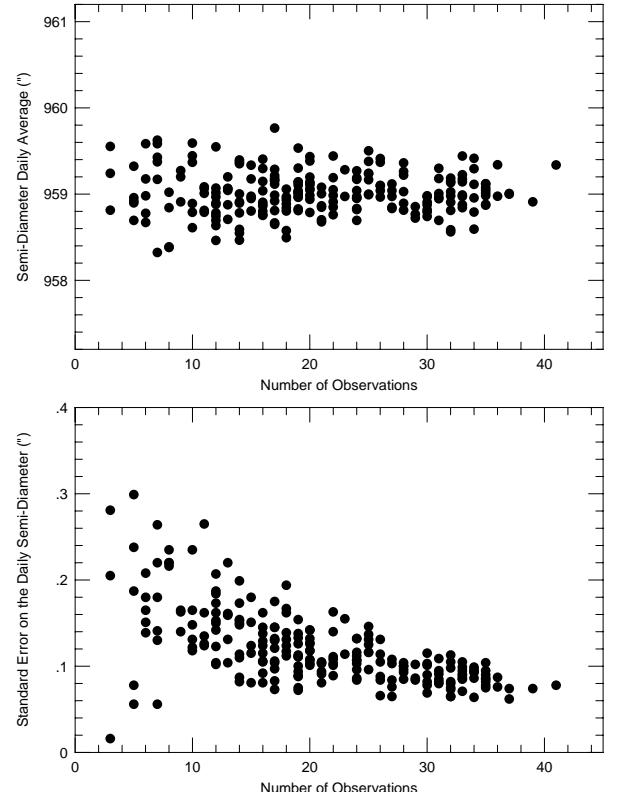

Fig. 2. Dependence of the daily average semi-diameter (top) and its standard error (bottom) on the number of observations used.
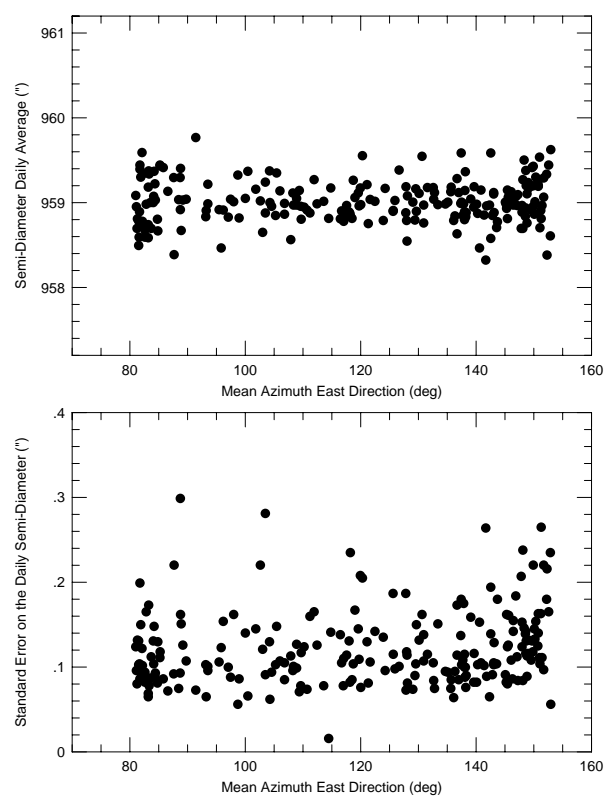

Fig. 3. Dependence of the daily average semi-diameter (top) and its standard error (bottom) on the mean azimuth direction.

24.5 and 31.8 days are regarded as associated with the solar rotation. A period of 49.1 days is commensurable with the lifetime of sunspots and faculae. These periods have also been found by Moussaoui et al. (2001), when analyzing the Calern observations. Strong periods of 67.5 , 77.1 and of 108.0 days appear and their origin is perhaps related to this particular data set or the solar activity in the observed dates. The 180 days period (which is the smallest one) is probably a residual artifact of atmospheric variations. 

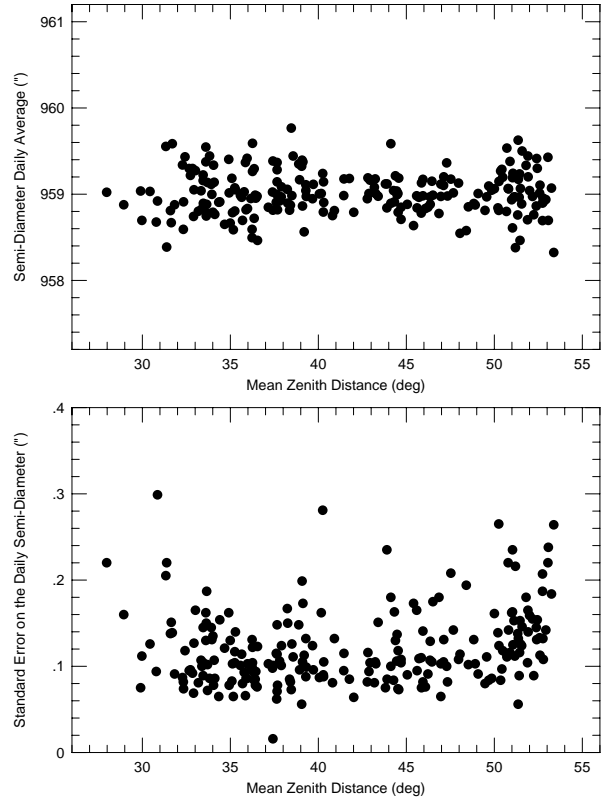

Fig. 4. Dependence of the daily average semi-diameter (top) and its standard error (bottom) on the mean zenith distance.
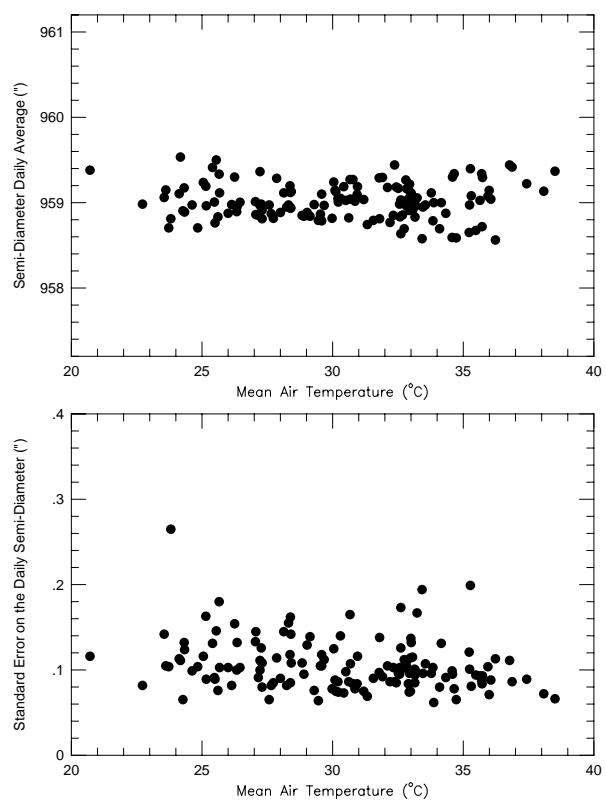

Fig. 5. Dependence of the daily average semi-diameter (top) and its standard error (bottom) on the mean air temperature.

\section{Conclusions}

Daily average values for the solar semi-diameter, observed at $5635 \AA$, with a modified solar astrolabe, are presented for the period from the beginning of July/1999 to the end of December/2000. From the 5031 independent measurements obtained in this period, a series of 225 daily points results. Each point generally comprises more than ten observations made at different azimuths, zenith distances, and along different heliolatitudes, as well as under different atmospheric conditions. In this way, the series is useful
Table 2. Amplitude spectrum for the semi-diameter series.

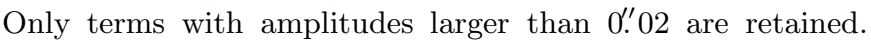
Neighbouring peaks closer than 10 days (Nyquist frequency chosen as 0.1) were sampled together.

\begin{tabular}{|c|c|}
\hline Period (days) & Amplitude $\left(^{\prime \prime}\right)$ \\
\hline 24.5 & 0.03 \\
31.8 & 0.03 \\
49.1 & 0.07 \\
67.5 & 0.06 \\
77.1 & 0.04 \\
108.0 & 0.04 \\
180.0 & 0.02 \\
\hline
\end{tabular}

to verify global variations of the solar radius, close to the maximum of cycle 23 .

The average value for the semi-diameter is $959^{\prime \prime} .01 \pm$ $0 . \prime 02$, with a standard deviation of 0.25 . The average standard error for the daily value is $0^{\prime \prime} 12$. There is no linear trend above 0 .' $008 /$ yr. However, there is weakly significant evidence of an increasing trend up the end of 1999, followed by decreasing results from the beginning of 2000 on.

Periodic terms with amplitudes between $0 .{ }^{\prime \prime} 02$ and $0 . \prime 07$ are present in the series. Periods commensurable with the solar rotation (24.5 and 31.8 days) and with the sunspot characteristic lifetime (49.1 days) are obtained. Longer periods $(67.5,77.1$ and of 108.0 days) seem to be related to this particular data series.

Acknowledgements. Part of this work was supported by $\mathrm{CNPq} /$ Brasil - CNRS/France binational agreement \#910172/95-1. E. Jilinski acknowledges MCT DTI grant \#381198/00-6 and FAPERJ grant \#E-26/152.221/2000. E. Reis Neto acknowledges the CNPq grant \#131957/2000-7 and FAPERJ grant \#E-26/150.856/2001.

\section{References}

Chollet, F., \& Sinceac, V. 1999, A\&AS, 139, 219

Golbasi, O., Chollet, F., Kiliç, H., et al. 2001, A\&A, 368, 1077 Irbah, A., Laclare, F., Borgnino, J., \& Merlin, G. 1994, Sol. Phys., 149, 213

Jilinski, E., Puliaev, S., Penna, J. L., et al. 1998, A\&AS, 130, 1

Jilinski, E., Puliaev, S., Penna, J. L., Andrei, A. H., \& Laclare, F. 1999, A\&AS, 135, 1

Kuhn, J. R., Bush, R. I., Scheick, X., \& Scherrer, P. 1998, Nature, 3, 92

Laclare, F., Delmas, C., \& Irbah, A. 1999, C.R. Acad. Sci. Paris, t. 327, série II b, 1107-1114

Moussaoui, R., Irbah, A., Fossat, E., et al. 2001, A\&A, 374, 1100-1107

Nöel, F. 2001, A\&A, 375, 614

Pap, J., Rozelot, J. P., Godier, S., \& Varadi, F. 2001, A\&A, 372, 1005

Puliaev, S., Penna, J. L., Andrei, A. H., \& Jilinski, E. 2000, A\&AS, 135, 1

Roberts, D. H., Lehár, J., \& Dreher, J. W. 1987, AJ, 93, 4

Sinceac, V., \& Chollet, F. 1999, A\&AS, 139, 219 